\title{
Canine Leishmaniosis: A Silent Burden in Southeast Asia and Elucidating Existing Knowledge Gap
}

\author{
Shaharul Nizim N, Watanabe M and Abdul Rani PA* \\ Department of Companion Animal Medicine and Surgery, Faculty of Veterinary \\ Medicine, Universiti Putra Malaysia, Malaysia
}

*Corresponding author: Dr. Puteri Azaziah Megat Abdul Rani, Department of Companion Animal Medicine and Surgery, Faculty of Veterinary Medicine, Universiti

\section{Research Article \\ Volume 4 Issue 1}

Received Date: February 11, 2019

Published Date: March 21, 2019

DOI: $10.23880 /$ oajvsr-16000172

Putra Malaysia, Malaysia, Tel: +603 8609 3896; 03 8609197; Email: azaziah@upm.edu.my

\begin{abstract}
Canine leishmaniosis is one of the most important canine vector-borne diseases (CVBD) caused by protozoan Leishmania $s p$. which pose zoonotic threat as some of its species are zoonotic. This parasitic disease is classified as Neglected Tropical Disease (NTD) and can be found in parts of the tropics, subtropics and southern Europe. A lot of studies had been carried out across the globe but lack in Southeast Asia, thus its epizootiology is still poorly understood. This article is meant to create awareness of the existence of this zoonotic disease amongst veterinary personnel, scientific community and readers.
\end{abstract}

Keywords: Leishmania; Southeast Asia; Zoonotic

Abbreviations: NTD: Neglected Tropical Disease; CVBD: Canine Vector-Borne Diseases; SEA: Southeast Asia; WHO: World Health Organization; CanL: Canine Leishmaniosis; VRI: Veterinary Research Institute; DAT: Direct Agglutination Tests.

\section{Introduction}

Neglected tropical diseases (NTD's) have caught global attention. Leprosy, lymphatic filariasis, visceral leishmaniosis and yaws are amongst NTD's continuously posing public health threat in eleven countries including Southeast Asia (SEA) region of the World Health Organization (WHO) [1]. Leishmaniosis, named after the Scottish pathologist William Leishman is caused by a group of haemoflagellate kinetoplastids. This neglected tropical disease are causing approximately 12 million to be infected with a species of Leishmania at times and its prevalence is up to at least in 98 countries and 3 territories on 5 continents [2]. In case of canine leishmaniosis (CanL), this disease are said to be endemic in more than 70 countries spreading all around the globe and affecting about 2.5 million dogs worldwide. Consequently, World Organization for Animal Health (OIE) has declared canine leishmaniosis (CanL) to be an important disease due to its zoonotic potential.

This haemoflagellates which are transferred to both humans and animals by phlebotomine sandflies are often neglected, as the disease caused by it is not in the priority list of national and international public health systems [3]. Nevertheless, the infections caused by this haemoparasite continuously moving across the globe and thrashing multi-host immunity systems and will likely to spread in 


\section{Open Access Journal of Veterinary Science \& Research}

forthcoming era as a silent killer and becoming silent burden especially in SEA where claimed to be nonendemic area. This is because this disease isn't a priority and often overlooked.

In Asia-Pacific, dog ownership is growing fast at a rate of $13 \%$ since the year 2006 until 2011. In 2011, the statistics showed that there are 62 million of households in Asia-Pacific owning a dog. Besides, based on the distributed questionnaires done by World Society for the Protection of Animals, dogs' population figure in 2007 in some of Asian and SEA are as shown in table 1.

\begin{tabular}{|c|c|}
\hline Countries & Figures \\
\hline Japan & 131,179000 \\
\hline Malaysia & 285,600 \\
\hline Indonesia & $8,000,000$ \\
\hline Nepal & 200000 \\
\hline Singapore & 107000 \\
\hline
\end{tabular}

Table 1: Dog's population in Asian and SEA.

Dogs have long been said to be responsible as the main animal reservoirs of Leishmania (Leishmania) infantum. It is well known that the dog plays an important role in the zoonotic cycle of transmission of L. (L.) infantum. For L. (V.) braziliensis, however, the dog is most likely to be an incidental host and its role in the zoonotic cycle of transmission is probably negligible [4].

Reithinger also claims that dogs where in several studies are said to be incriminated as primary vector of zoonotic visceral leishmaniosis, is just a circumstantial evidence. Nonetheless, it has been put forward that by targeting infected dogs could lessen the case of American cutaneous leishmaniosis [5]. A study was conducted to elucidate the role of domestic dogs in transmission of visceral leishmaniasis in Eastern Sudan; interestingly it is found out that dogs seem to play role in the dynamics of visceral leishmaniosis transmission based on serology and molecular result. However, additional imperative works are indicated to reflect a genuine infection rate [6] Leishmania requires two hosts to complete its life cycle, flagellated infective promastigote form is transmitted by a female phlebotomine sand fly, and the intracellular amastigote from need a mammal host to develop and replicate. To date, sandflies are the only known vector that able to transmit this parasitic disease biologically. Solano in his paper also explains that even in presence of low numbers of sandflies $(0.5-3 \%)$, it is sufficient for the disease to remain stable in endemic areas. Non-sand fly modes of transmission have also been described but their role in the natural history and epidemiology of leishmaniosis remains unclear

Many species of Leishmania have been described and the ones that play significant role in medical and veterinary world are about 10 , which most of them are zoonotic [7]. About 800 phlebotomine sand fly species estimated to exist, about 98 species are currently proven or suspected vectors of leishmaniasis, and these include 42 Phlebotomus species in the Old World and 56 Lutzomyia species in the New World [8]. Sandflies generally live in tropical and subtropical regions and this is because sandflies are found in various habitats, ranging from semi-desert to rainforest $[9,10]$.

\section{Canine Leishmaniosis Vector: An Abundance in SEA}

In Thailand, Apiwathnasorn conducted a survey on Phlebotomine sand flies and it is found out that sandflies can be found in caves, termite hills, abandoned house, ancient atone sanctuaries, air-raid shelters, tree hollows, loose bark of dead standing trees and rock crevices[11]. Later in July 2014, a study was conducted to gain better knowledge on the reservoirs and vectors of L. siamensis that have emerged in southern Thailand. They captured 378 female and 110 male sandflies and were tested for Leishmania DNA. Interestingly, Leishmania DNA amplicon was identified in two species of female sandflies, Sergentomyia (Neophlebotomus) and Sergentomyia (Parrotomyia) barraudi [12].

In Malaysia, a study was conducted to reveal the population status of phlebotomine sand fly in lime stone areas and caves of Western Malaysia. It is found out that sand flies species are found in abundance where 1,548 specimens from 18 sand fly species were collected [13]. In July 2016, a study done by Azian, et al. in Malaysia where 1281 sand flies were caught and microscopically identified, it was found that 981 were Phlebotomus spp and 237 were Sergentomyia spp. None of the sandflies were positive for Leishmania spp by both microscopic examination and PCR. Their study revealed that even though the seroprevalence of leishmaniosis among immigrant workers were relatively high, the vectors were all tested negatives [14]. In another parts of SEA, there is lack of study on phlebotomine sand fly population, therefore, not much information available online for reference.

It is curious to know the status of vector population in other SEA countries, where the infections are reported 
but sandflies as traditional vectors has never been investigated. Canine leishmaniosis: Cases reported in SEA countries Veterinary research institute (VRI) in Malaysia has been committed in studying blood parasite infection in dogs. Hence, 103 pet dogs around Ipoh were screened for common blood protozoa and Leishmania, the neglected tropical disease which is identified using PCR; however, no dogs were tested positive for leishmaniosis [15].
In Thailand, cats and cows are amongst the species where Leishmania antibody was found using Leishmania antibody Positive direct agglutination tests (DAT). However, no study has been done to prove dogs as potential reservoirs [16]. For the remaining SEA countries, canine leishmaniosis status still remains unclear since there is no available data. Table 2 shows the comparison of recorded cases of human leishmaniosis and canine leishmaniosis in SEA.

\begin{tabular}{|c|c|c|c|c|c|}
\hline Countries & Reported human cases & Reported canine cases & Countries & Reported human cases & $\begin{array}{c}\text { Reported } \\
\text { canine cases }\end{array}$ \\
\hline Malaysia & $\begin{array}{c}\text { Few reported cases from } \\
\text { immigrants who came } \\
\text { rom endemic area }[17,18]\end{array}$ & $\begin{array}{c}\text { No case reported, but there } \\
\text { is anecdotal evidence from } \\
\text { private practitioner } \\
\text { encounter dogs have } \\
\text { similar clinical signs }\end{array}$ & Thailand & $\begin{array}{c}\text { Endemic cutaneous } \\
\text { leishmaniosis (WHO, 2017) } \\
\text { First isolation of leishmania in } \\
\text { local people [19] }\end{array}$ & No report \\
\hline Brunei & No report (Viroj, & No report & Laos & No report & No report \\
\hline
\end{tabular}

Table 2: Comparison of recorded cases of human and canine leishmaniosis in SEA region.

\section{Leishmaniosis: Silent Burden in SEA Region}

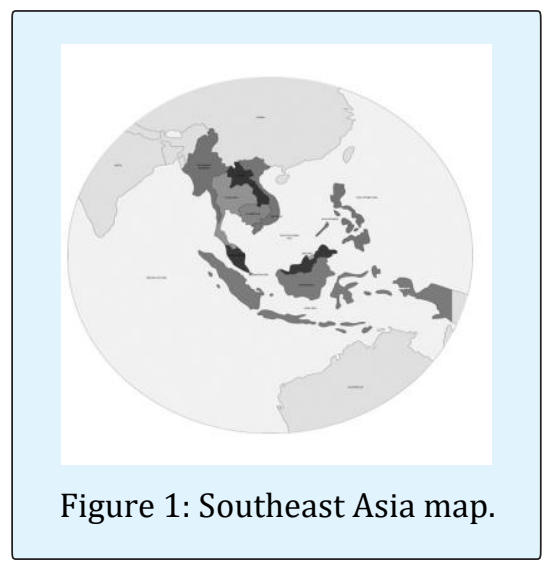

In Malaysia, human leishmaniosis were reported in immigrant workers. Both of the cases took longer investigation period before coming up with diagnosis because leishmaniosis was failed to be listed as initial differential diagnosis. Vigorous treatment given preleishmania diagnosis but condition of the patients worsen, fortunately eventually definitive diagnosis as visceral leishmaniosis was able to be concluded and appropriate treatment was given and patients recovered. These scenarios indicate the importance of awareness among treating medical personnel on this neglected tropical diseases. In Malaysian veterinary community, there is anecdotal evidence of private practitioner encountering dogs having the same clinical signs as canine leishmaniosis. However, to date, no canine leishmaniosis has been reported from the veterinarian. Hence, literacy of local treating veterinarian on this neglected disease need to be improved so that the chances of putting canine leishmaniosis in probable diagnosis is no longer slim to narrow.

Geographically, northwest Peninsular Malaysia which are Kedah and Perlis share international borders with provinces of Thailand. Thailand on the other hand has high prevalence with leishmaniosis according to World Health Organisation (WHO). The chances of leishmaniosis in both human and dogs to communicate are made possible through this porous border and the status of leishmaniosis in the local people and dogs remain questionable and mysterious.

In Thailand since 1996, four autochthonous cases were reported and the first report of autochthonous visceral leishmaniosis in HIV-infected Thai. This patient has never travelled outside except worked as fisherman in Indian Ocean and Northern Indonesia where no leishmaniosis has ever been reported in Indonesia [20]. An investigation on potential animal reservoirs had been carried out in effected Thailand provinces where autochothonous leishmaniosis has been described. It is found out that Leishmania DNA was detected in black rats where their blood, liver and spleen were sampled [12]. Besides, in another study, antibody against leishmaniosis was also detected in 9 cats [16]. However, despite having reported human cases, and Leishmania DNA and antibody in rats and cats respectively, none of the case is reported 
in dogs. With an increasing number of patients with autochthonous leishmaniasis in association with the presence of potential vector, it remains to be investigated whether this vector-borne disease are able to pose disease threat in local Thailand dogs as dogs are the main reservoir of this disease.

Vietnam shares its border with seven other Asian countries, where the border stretches from China to the north, Laos to the northwest, Cambodia to the southwest, Thailand across the Gulf of Thailand to the southwest, and the Philippines, Malaysia and Indonesia across the South China Sea to the east and southeast. Leishmania expansion has gone beyond its natural ecotopes, it could now be found where it was naïve before [21]. Poor countries like India and East Africa score high prevalence of this disease but leishmaniasis was not considered an endemic disease in SEA [22].

Surprisingly, leishmaniosis is expanding across the globe into new geographic regions. Reports from Vietnam highlight the emergence of leishmaniosis in areas of Asia where it was not previously found.

According to the Asia Canine Protection Alliance, more than 5 million dogs were sacrificed annually for meat in Vietnam. This scenario has resulted up to half a million dogs a year to be imported into Vietnam across their border point to meet their self-sufficiency level. Despite meat, infectious diseases are also brought in through this crossing point because almost all of the dogs aren't vaccinated and do not have proper documentation. This practice is alarming in that area because it poses significant risk to human health and disease control. In 2009, Rosypal in his study suggested that their local dogs are likely not involved in the emergence of visceral leishmaniosis in three HIV infected women. This is proven through serology work done to test Leishmania antibody using immunochromatographic dipstick assay [23].

Indeed, leishmaniosis has been reported in many regions of the world where it was not found previously [21]. Economically poor communities in parts of East Africa and India are particularly affected by VL [22], but leishmaniosis was not considered an endemic disease in SEA. Surprisingly, leishmaniosis is expanding across the globe into new geographic regions. Reports from Vietnam, and a subsequent case of indigenous leishmaniosis in Taiwan, highlight the emergence of leishmaniosis in areas of Asia where it was not previously found.

To date, none of leishmaniosis has ever been reported in Myanmar, even though it is geographically bordered by
India where leishmaniosis is highly endemic. This case does not only to happen in human but also animal reservoir including dogs where dogs were found to be positive with $\mathrm{Rk} 39$ serology that indicates visceral leishmaniosis [24]. Even in India where leishmaniosis is found to be endemic, the role of dogs as potential revoir hasn't carefully explored [25].

East Timor and Singapore have had leishmaniosis cases reported in human but not in dogs. Of interest, local people from these countries aren't spared but possibility of animal reservoir has never been identified. While in Cambodia, Laos and Brunei have never reported any human and canine leishmaniosis case [26,27].

It is unfortunate for countries in SEA to not have a proper scientific evidence of dogs' role as Leishmania reservoir which in our standpoint it is under reported despite of having large population of stray dogs. Vigorous epidemiological studies on canine leishmaniosis urgently needed to be initiated in these countries to unravel the real silent status of canine leishmaniosis in SEA.

\section{Conclusion}

Canine leishmaniosis should be given due attention in SEA as it could have been overlooked and remain unexpressed all these while. Due to globalization, where mode of transportation made ease and communication of infections are made possible, veterinarians should be literate in recognising these neglected tropical disease (NTD'S) which will eventually help to unravel the real disease burden, implementation of public health policy and lead to better cure rates.

\section{References}

1. Narain JP, Dash AP, Parnell B, Bhattacharya SK, Barua $S$, et al. (2010) Elimination of neglected tropical diseases in the South-East Asia Region of the World Health Organization. Bull World Health Organ 88: 206-210

2. Alvar J, Vélez ID, Bern C, Herrero M, Desjeux P, et al. (2012) Leishmaniasis worldwide and global estimates of its incidence. PLoS ONE 7(5): e35671.

3. Moriconi M, Rugna G, Calzolari M, Bellini R, Albieri A, et al. (2017) Phlebotomine sand fly-borne pathogens in the Mediterranean Basin: Human leishmaniasis and phlebovirus infections. PLoS Neglected Tropical Diseases 11(8): 1-19. 
4. Dantas-Torres F (2007) The role of dogs as reservoirs of Leishmania parasites, with emphasis on Leishmania (Leishmania) infantum and Leishmania (Viannia) braziliensis. Veterinary Parasitology 149(3-4): 139146.

5. Reithinger R, Davies CR (1999) Is the domestic dog (Canis familiaris) a reservoir host of American cutaneous leishmaniasis? A critical review of the current evidence. Am J Trop Med Hyg 61(4): 530-541.

6. Hassan MM, Osman OF, Fathi MA El-Raba'a, Schallig HD, Elnaiem DEA (2009) Role of the domestic dog as a reservoir host of Leishmania donovani in eastern Sudan. Parasites and Vectors 2(1): 1-7.

7. Bates PA (2007) Transmission of Leishmania metacyclic promastigotes by phlebotomine sand flies. International Journal for Parasitology 37(10): 10971106.

8. Maroli M, Feliciangeli MD, Bichaud L, Charrel RN, Gradoni L (2013) Phlebotomine sandflies and the spreading of leishmaniases and other diseases of public health concern. Med Vet Entomol 27(2): 123147.

9. Koch LK, Kochmann J, Klimpel S, Cunze S (2017) Modeling the climatic suitability of leishmaniasis vector species in Europe. Scientific Reports 7(1): 110.

10. Rozendaal JA (1997) Mosquitos and other biting Diptera. Vector Control: Methods for Use by Individuals and Communities. World Health Organization, pp: 6-28.

11. Apiwathnasorn C, Sucharit S, Rongsriyam Y, Leemingsawat S, Kerdpibule V, et al. (1989) A brief survey of phlebotomine sandflies in Thailand. Southeast Asian J Trop Med Public Health 20(3): 429432.

12. Chusri S, Thammapalo S, Silpapojakul K, Siriyasatien $P$ (2014) Animal reservoirs and potential vectors of Leishmania siamensis in southern Thailand. Southeast Asian J Trop Med Public Health 45(1): 1319.

13. Shahar MK, Hassan AA, Lee HL, Salmah MRC (2011) Studies of phlebotomine sand fly (Diptera: Psychodidae) populations in Limestone areas and caves of Western Malaysia. Southeast Asian Journal of Tropical Medicine and Public Health 42(1): 83-93.
14. Noor Azian MY, Lokman Hakim S, Khadri MS, Yusri MY, Adela JI, et al. (2016) Leishmaniasis in Peninsular Malaysia: The role of immigrant workers and the vector. Southeast Asian Journal of Tropical Medicine and Public Health 47(4): 607-616.

15. Jamnah O, Chandrawathani P, Premaalatha B, Zaini CM, Sheikh Abdul Muizz SI, et al. (2016) Blood Protozoa Findings in Pet Dogs Screened in Ipoh , Malaysia. Malaysian Journal of Veterinary Research 7(1): 9-14.

16. Sukmee T, Siripattanapipong $\mathrm{S}$, Mungthin $\mathrm{M}$, Worapong J, Rangsin R, et al. (2008) A suspected new species of Leishmania, the causative agent of visceral leishmaniasis in a Thai patient. International Journal for Parasitology 38(6): 617-622.

17. Ab Rahman AK, Abdullah FH (2011) Visceral leishmaniasis (kala-azar) and malaria coinfection in an immigrant in the state of Terengganu, Malaysia: A case report. J Microbiol Immunol Infect 44(1): 72-76.

18. Hamidah NH, Cheong SK, Abu Hassan J (1995) A case of kala-azar diagnosed by bone marrow aspiration. The Malaysian Journal of Pathology 17(1): 39-41.

19. Pothirat $T$, Tantiworawit A, Chaiwarith $R$, Jariyapan $\mathrm{N}$, Wannasan A, et al. (2014) First Isolation of Leishmania from Northern Thailand: Case Report, Identification as Leishmania martiniquensis and Phylogenetic Position within the Leishmania enriettii Complex. PLoS Neglected Tropical Diseases 8(12): e3339.

20. Suankratay C, Suwanpimolkul G, Wilde H, Siriyasatien P (2010) Case report: Autochthonous visceral leishmaniasis in a human immunodeficiency virus (HIV)-infected patient: The first in Thailand and review of the literature. Am J Trop Med Hyg 82(1): 48.

21. Shaw J (2007) The leishmaniases - Survival and expansion in a changing world. A mini-review. Mem Inst Oswaldo Cruz 102(5): 541-547.

22. Chappuis F, Sundar S, Hailu A, Ghalib H, Rijal S, et al. (2007). Visceral leishmaniasis: What are the needs for diagnosis, treatment and control? Nat Rev Microbiol 5(11): 873-882.

23. Rosypal AC, Hailemariam S, Wekheye V, Huong LT, Dubey JP, et al. (2009) Survey of Dogs From Vietnam 
for Antibodies to Visceralizing Leishmania spp. J Parasitol 95(3): 767-767.

24. Singh N, Mishra J, Singh R, Singh S (2013) Animal Reservoirs of Visceral Leishmaniasis in India. J Parasitol 99(1): 64-67.

25. Megat Abd Rani PA, Irwin PJ, Gatne M, Coleman GT, Traub RJ (2010) Canine vector-borne diseases in India: a review of the literature and identification of existing knowledge gaps. Parasites \& Vectors 3(1): 28.
26. Viroj W (2012) Leishmaniasis in Southeast Asia: The story of the emergence of an imported infection in a non-endemic area of the world. Journal of the University of Malaya Medical Centre 15(1): 1-4.

27. Chevalier B, Carmoi T, Sagui E, Carrette P, Petit D, et al. (2000) Report of the First Cases of Cutaneous Leishmaniasis in East Timor. Clinical Infectious Diseases 30(5): 840-840. 